\title{
Abundance Signatures in Halo Stars: Clues to Nucleosynthesis in the First Stars
}

\author{
John J. Cowan*, Christopher Sneden ${ }^{\dagger}$, James E. Lawler and Elizabeth A. Den \\ Hartog** and Jason Collier*
}

* Homer L. Dodge Department of Physics \& Astronomy, University of Oklahoma, Norman, OK 73019

${ }^{\dagger}$ Department of Astronomy and McDonald Observatory, University of Texas, Austin, TX 78712

** Department of Physics, University of Wisconsin, Madison, WI 53706

\begin{abstract}
.
We are using both space-based (Hubble Space Telescope, HST) and ground-based telescopes to make extensive studies of Galactic halo stars. These stars contain the nucleosynthesis products (from the rapid neutron capture process, i.e., the $r$ process) from the earliest generations of stars - the progenitors of the halo stars. The observed stellar abundance distributions - from the lightest neutron-capture elements, such as Ge, along with some of the heaviest, including $\mathrm{Pt}$ - are providing new clues about the earliest Galactic $r$-process nucleosynthesis. These in turn will help to identify the characteristics and nature of the first stars in the Galaxy.
\end{abstract}

Keywords: atomic spectra; elemental abundances in stars; nucleosynthesis: stellar PACS: $32.30 ; 97.10 . \mathrm{Tk} ; 97.10 . \mathrm{Cv}$

\section{INTRODUCTION}

Halo stars contain the nucleosynthetic "signatures" from the very first stars in our Galaxy. Thus, the nature and quantities of the elements observed in the metal-poor halo stars provide direct insight into the types of nucleosynthetic processes that must have occurred early in the history of the Galaxy. Since these nuclear formation processes are also dependent upon stellar masses, the observed stellar abundance distributions also provide clues about the masses of the first stars - the progenitors of the halo stars.

\section{ABUNDANCES OF N-CAPTURE ELEMENTS IN METAL-POOR HALO STARS}

There have been extensive observations of the Galactic halo stars over the last several decades. In particular there have been many studies of the elemental abundance distributions of the neutron capture elements - i.e., those made in either the slow ( $s-$ ) or rapid $(r-)$ neutron-capture processes - in these stars (see, e.g., $[1,2,3]$ for recent reviews).

\section{Abundances and Atomic Data}

The advances in abundance studies - that are providing new insights into the nature of neutron capture nu- cleosynthesis in the initial burst of Galactic nucleosynthesis - are being driven by improvements in both observational data and in laboratory atomic data. We show in Figure 1 abundance comparisons for three halo stars, with the abundances normalized at Eu. Plotted are differences between the abundance determinations, based upon older, published atomic data, and the solar system $r$-process only values. It is seen that there is significant star-to-star scatter and that the elemental, stellar abundances deviate from the solid horizontal line, indicating solar system $r$-process only values.

We show the same comparisons in Figure 2, but now with recent new experimental data (improved laboratory transition probabilities) for Nd [4], Sm [5], Gd [6] and Ho [7]. It is seen that the scatter has been significantly reduced and that the abundances are consistent from starto-star and with the solar system $r$-process only values. New transition probabilities have also recently been obtained for the element Hf [8], and additional elemental and isotopic studies are underway.

\section{Stellar Abundance Signatures}

We show in Figure 3 comparisons of neutron-capture abundances in five $r$-process-rich Galactic halo stars: CS 22892-052 [9], HD 115444 [10], BD +17 3248 [11], CS 31082-001 [12], and HD 221170 [13]. The solid lines are the scaled $r$-process only solar system abundance curves [14]. The comparisons clearly indicate that all five of the stars show similar abundance patterns - an 


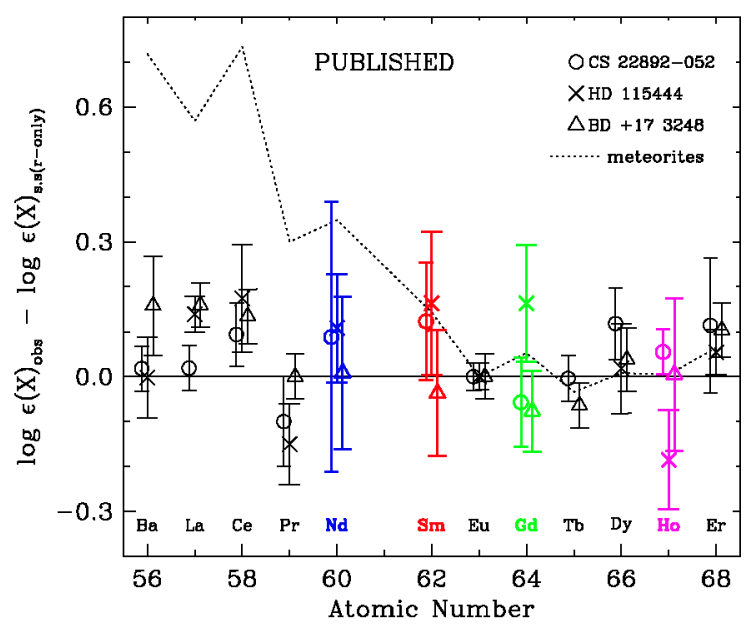

FIGURE 1. Differences between neutron-capture elemental abundances for three halo stars and the Sun based upon older atomic data. Differences equal to zero, lying on the solid horizontal line, indicate perfect agreement with solar system $r$ process only predictions.

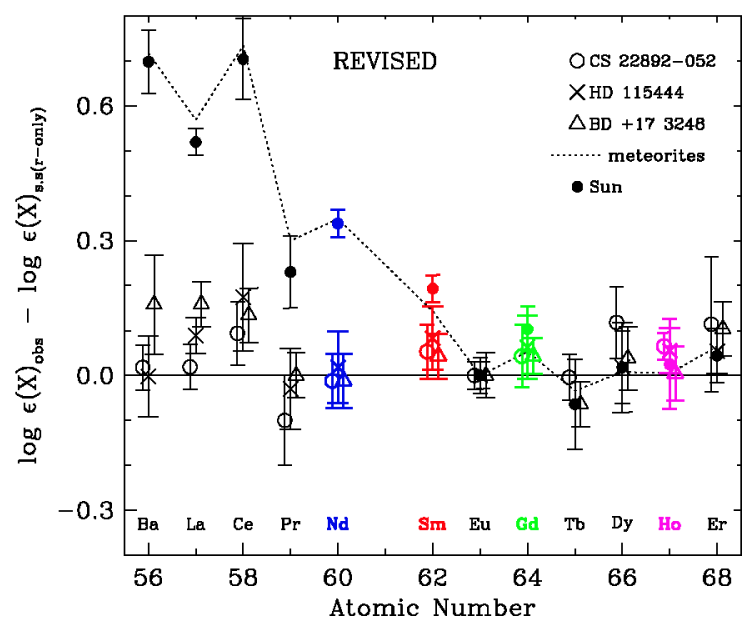

FIGURE 2. Same as Figure 1, except the abundances now based upon newer experimental atomic data. Total solar system, $s$ - plus $r$-process abundances, are also shown (from $[7,5,4,6]$.

agreement with the solar system $r$-process only curve for the elements $\mathrm{Ba}(Z=56)$ and above. This agreement strongly suggests a robust $r$-process working over billions of years from the early history of the Galaxy when the halo stars were formed - until the formation of the Sun. This further suggests a unique $r$-process site or at least a well constrained astrophysical environment for the formation of these heavy neutron capture elements. Examination of Figure 3 indicates some deviations between the solar curve and the stellar data for the lighter

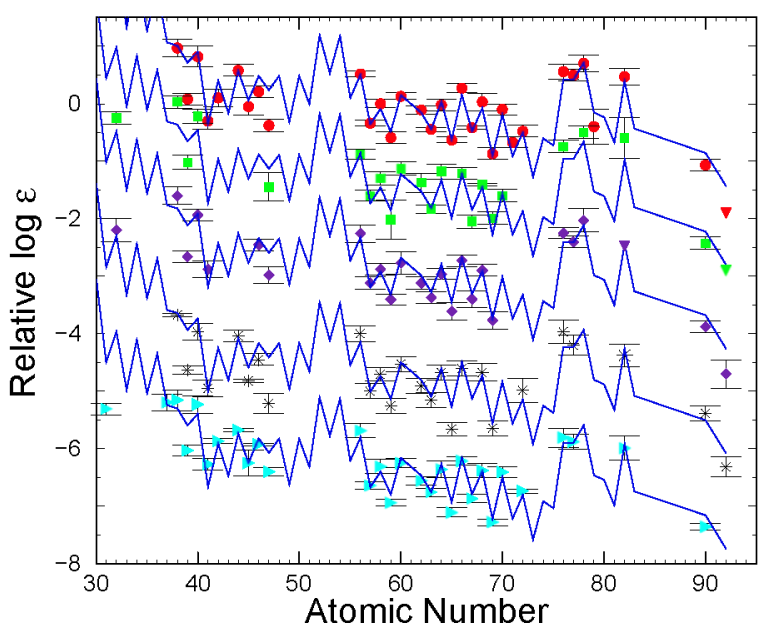

FIGURE 3. Comparisons of neutron-capture abundances in five $r$-process-rich Galactic halo stars: starting from the top CS 22892-052 (circles), HD 115444 (squares), BD +17 3248 (diamonds), CS 31082-001 (stars), and HD 221170 (rightfacing triangles). The solid lines are the scaled $r$-process only solar system elemental abundance curves (after [15]).

n-capture elements. This has led to suggestions of a second (or even multiple) $r$-process sites (see [15] for further discussion).

\section{ABUNDANCE SCATTER IN THE GALAXY: EARLY GALACTIC NUCLEOSYNTHESIS}

We show in Figure 4 the variation of $[\mathrm{Eu} / \mathrm{Fe}][16]$ and $[\mathrm{Mg} / \mathrm{Fe}][17]$ as function of metallicity for a sample of halo and disk stars. It is seen that at low metallicities (and early Galactic times) there is a very large scatter in the $[\mathrm{Eu} / \mathrm{Fe}]$ abundances. With increasing metallicity (and later times) this scatter disappears. These data suggest an early unmixed (chemically inhomogeneous) Galaxy in neutron capture elements. It further suggests that $r$-process production was rare in the early Galaxy that only a few stars synthesized elements such as Eu, and that iron production was not strongly coupled to $r$ process production.

The $[\mathrm{Mg} / \mathrm{Fe}]$ data [17] exhibit a different pattern. (We show selected data for metal-poor stars only. A more comprehensive compilation will appear in [18]. There is little scatter in these data at low metallicities, though on average the abundances lie above the solar value indicated by the solid horizontal line. These $[\mathrm{Mg} / \mathrm{Fe}]$ data suggest that $\mathrm{Mg}$ production was common in the early Galaxy and the types of stars that were synthesizing Fe were also synthesizing Mg. It is well-known that massive 


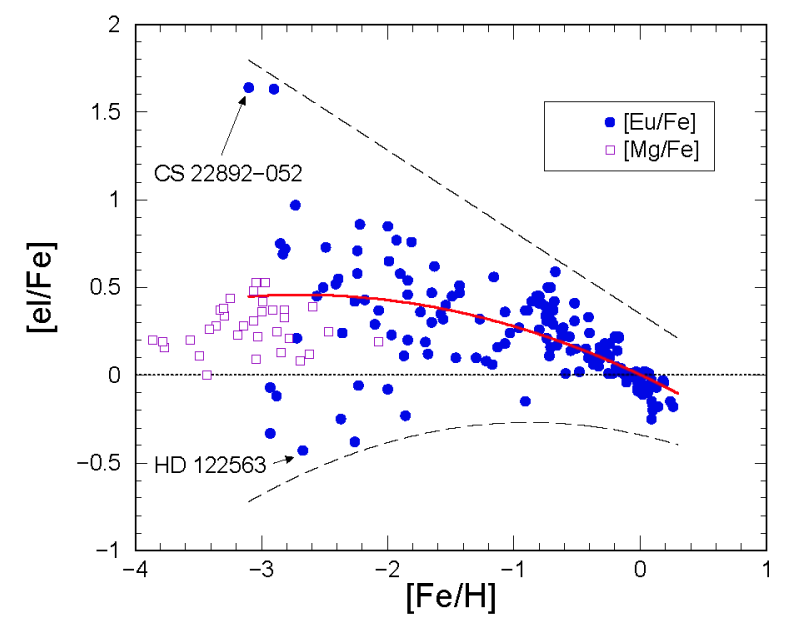

FIGURE 4. Eu/Fe (filled circles) for a sample of halo and disk stars from [16]. Representative $\mathrm{Mg} / \mathrm{Fe}$ data (open squares), for metal-poor stars only, from [17]. The solid line is a leastsquare fit to the Eu data, the dotted line indicates a solar value and the two dashed lines indicate the extent of the $\mathrm{Eu} / \mathrm{Fe}$ data.

stars - stars that become core-collapse supernovae are responsible for the formation of $\alpha$-elements, such as $\mathrm{Mg}$. Thus, the abundance comparisons illustrated in Figure 4 suggest different mass ranges and different sites for the synthesis of the $\alpha$-elements and the neutroncapture elements. There have been previous suggestions that the $r$-process site might be in lower mass supernovae (i.e., $\simeq 8-10 \mathrm{M}_{\odot}$ ) and not in the more massive stars ( $20 \mathrm{M}_{\odot}$ ) responsible for the formation of the $\alpha$-elements. The data illustrated here are consistent with such an interpretation.

\section{SUMMARY}

Abundance observations indicate the presence of neutron capture (i.e., $s$ - and r-process) elements in old Galactic halo stars. These observations provide insight into the nature of the earliest generations of stars in the Galaxy - the progenitors of the halo stars - responsible for neutroncapture synthesis. Specifically, observational and theoretical comparisons of the elemental abundances in $r$ process rich and $r$-process poor halo stars are providing clues about the extent of, and the types of stars involved in, heavy element nucleosynthesis early in the Galaxy. These stellar abundance determinations depend critically upon atomic data. In particular improved laboratory transition probabilities have been recently obtained for a number of elements. This has allowed for more reliable determinations of the abundances in metal-poor Galactic halo stars. Abundance comparisons among the $r$-processrich halo stars - employing the newly determined stellar abundances - show that the heaviest neutron-capture elements (i.e., $\mathrm{Ba}$ and above) are consistent with a scaled solar system $r$-process abundance distribution, while the lighter neutron-capture elements do not conform to the solar pattern. These comparisons suggest the possibility of two $r$-process sites in stars. We also observe a large star-to-star scatter in the observed abundances of neutron-capture element/iron ratios at low metallicities which disappears with increasing metallicity or $[\mathrm{Fe} / \mathrm{H}]-$ suggesting the formation of these heavy elements (presumably from certain types of supernovae) was rare in the early Galaxy.

\section{ACKNOWLEDGMENTS}

We thank our colleagues for valuable insights and contributions. This work has been supported in part by NSF grants AST 07-07447 (J.J.C.), AST 06-07708 (C.S.), AST 05-06324 (J.E.L. and E.A.D.H.), and by STScI.

\section{REFERENCES}

1. C. Sneden and J. J. Cowan, Science 85, 2503-2504 (2003).

2. J. W. Truran, J. J. Cowan, C. A. Pilachowski and C. Sneden, PASP 114, 1293-1308 (2002).

3. J. J. Cowan and C. Sneden, Nature 440, 1151-1156 (2006).

4. E. A. Den Hartog et al., ApJS 148, 543-566 (2003).

5. J. E. Lawler et al., ApJS 162, 227-260 (2006).

6. E. A. Den Hartog et al., ApJS 167, 292-314 (2006).

7. J. E. Lawler et al., $A p J \mathbf{6 0 4}, 850-860$ (2004).

8. J. E. Lawler et al., ApJS 169, 120-136 (2007).

9. C. Sneden et al., $A p J$ 591, 936-953 (2003).

10. J. Westin et al., $A p J$ 530, 783-799 (2000).

11. J. J. Cowan et al., $A p J$ 572, 861-879 (2002).

12. V. Hill et al., $A \& A$ 387, 560-579 (2002).

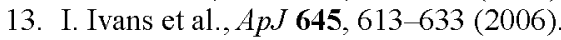

14. J. J. Cowan et al., "Nucleosynthesis: Stellar and Solar Abundances and Atomic Data," in Proceedings of the 2006 NASA Laboratory Astrophysics Workshop, edited by P. F. Weck, V. Kwong, \& F. Salama, NASA, Washington, DC, 2006 , pp. 82-87.

15. K.-L. Kratz et al., ApJ 662, 39-52 (2007).

16. J. J. Cowan and F.-K. Thielemann, Physics Today 57, 47-53 (2004)

17. R. Cayrel et al., $A \& A 416,1117-1138$ (2004).

18. C. Sneden, J. J. Cowan, \& R. Gallino, in prep. (2008). 